\title{
The Relationship Between Individual Differences in Intraindividual Variability in Core Affect and Interpersonal Behaviour
}

\author{
TINNEKE TIMMERMANS ${ }^{1 *}$, IVEN VAN MECHELEN ${ }^{1}$ and PETER KUPPENS ${ }^{1,2}$ \\ ${ }^{1}$ Department of Psychology, University of Leuven, Belgium \\ ${ }^{2}$ Department of Psychology, School of Behavioural Science, University of Melbourne, Australia
}

\begin{abstract}
How people's feelings and interpersonal behaviour change across time can be represented as movements within a core affect and an interpersonal space. To gain insight into the relationship between affect and behaviour dynamics, the present study examined how individual differences in intraindividual variability in core affect relate to those in interpersonal behaviour, and how both are related to personality traits. In an experience sampling study, 63 participants were asked to monitor their core affect during one week and their interpersonal behaviour during another one. The results demonstrated a fairly consistent correspondence between several indices of people's variability in core affect and interpersonal behaviour, indicating that emotional lability also signals behavioural volatility and vice versa. Copyright (C) 2010 John Wiley \& Sons, Ltd.
\end{abstract}

Key words: experience sampling; core affect; interpersonal behaviour; intraindividual variability; personality

\section{INTRODUCTION}

Psychology is concerned with both covert and overt aspects of psychological functioning. Covert aspects include, among others, the feelings and core affect people experience, and overt aspects include people's interpersonal behaviour. Core affect and interpersonal behaviour are clearly distinct and reflect different psychological phenomena. Yet, this does not preclude that these covert and overt levels of psychological functioning are related.

The essence of people's covert feelings and overt interpersonal behaviour can be captured by means of different sets of properties or dimensions. In the present paper, we focus on two-dimensional theoretical proposals identifying the dimensional nature of affect and interpersonal behaviour (although other proposals exist as well: e.g. Schimmack \& Grob, 2000). In terms of affect, the most basic characteristics of people's feelings, emotions and

*Correspondence to: Tinneke Timmermans, Department of Psychology, KULeuven, Tiensestraat 102, 3000 Leuven, Belgium. E-mail: tinneke.timmermans@psy.kuleuven.be 
other affective experiences can be described in terms of pleasure (ranging from pleasure through a neutral point to displeasure) and arousal (ranging from sleep, then drowsiness, through various stages of alertness to extreme excitement). These properties combine to form core affect, a 'neurophysiological state consciously accessible as the simplest raw (nonreflective) feelings evident in moods and emotions' (Russell, 2003, p. 148).

Interpersonal behaviours can also be organized as a function of two fundamental underlying properties, namely agency and communion (Wiggins, 1979, 1991). Agentic behaviours can be conceptualized as behaviours that assert status relative to others whereas communal behaviours can be conceptualized as behaviours that promote interpersonal ties. Agency is represented by a bipolar axis ranging from assertive-dominant to passivesubmissive behaviour. Communion is represented by a bipolar axis ranging from agreeable to quarrelsome behaviour (Wiggins, 1991).

Both people's core affect and interpersonal behaviour vary across time and circumstances, and these changes can be represented as movements within the core affect and interpersonal two-dimensional space, respectively. These movements can take different forms, and individuals can differ in several crucial characteristics, such as their mean position (how people feel and behave on average) and amount of intraindividual variability displayed (how much people's feelings and behaviours change across time; Kuppens, Van Mechelen, Nezlek, Dossche, \& Timmermans, 2007; Moskowitz \& Zuroff, 2004, 2005a).

Understanding how these individual differences in intraperson variability in core affect and interpersonal behaviour are interrelated can provide a gateway to obtain insight into the relationships between core affect and interpersonal behaviour. More specifically, there is an interesting possibility of a correspondence between people's variability in interpersonal behaviour and affect. In other words, variability in how agreeable or dominant one behaves towards others may be related to variability in how pleasant or active one feels. In general, there are strong theoretical reasons for postulating an inherent link between emotions and interpersonal behaviour. Contemporary perspectives on emotions see the function of emotions in motivating behaviour to maintain or change the individual's relation to his or her social environment (e.g. Barrett, 1998; Frijda, 1986; Scherer, 2009). To achieve this goal, emotions are thought to consist of different components that inform the individual of his or her relationship with the social environment, and consequently motivate the individual to action when threats or opportunities arise. In this view, a crucial role is played by action tendencies, which are thought to form the link between emotion and action and lie at the basis of processes that instigate behaviour (e.g. Frijda \& Mesquita, 1994). On the other hand, embodiment theories of emotions clearly argue that how people behave and act deeply affects their emotional responses as well (Niedenthal, 2007). As a result, emotion and behaviour are thought to be intricately linked: How we feel reflects on how we behave, and how we behave reflects on how we feel.

\section{Intraindividual variability}

Researchers have developed different measures of intraindividual variability in the respective two-dimensional spaces. Flux refers to variability about an individual's mean score on a separate dimension of core affect (Kuppens et al., 2007) or interpersonal behaviour (Moskowitz \& Zuroff, 2004, 2005a). To operationalize flux the standard deviation about the mean is calculated. Pulse and spin are linked to the circular geometry of the core affect and the interpersonal circumplex. Core affect and interpersonal behaviour within an interaction may be represented as a vector extending from the origin to a point in the space and defined with respect to the coordinates (i.e. the polar coordinates of that position) of vector length and 
angular displacement. To operationalize pulse, variability in terms of intensity, and spin, variability in the qualitative nature of positions, we relied on these coordinates. The length reflects the intensity of experienced core affect or interpersonal behaviour whereas the angle reflects the quality. Pulse and spin are illustrated in Figure 1. It is important to emphasize that pulse and spin are independent from each other: Fluctuations in the intensity of feelings or interpersonal behaviour vary regardless of their quality.

\section{Relationships between individual differences in intraindividual variability in affect and behaviour}

Interindividual differences in flux, pulse and spin were studied by Moskowitz and Zuroff (2004, 2005a) with regard to interpersonal behaviour, and by Kuppens et al. (2007) with regard to core affect. In both studies the relation between individual differences in these intraperson variability scores and several dispositional variables were examined. When comparing both studies, it is remarkable how similar dispositional variables relate to individual differences in flux, pulse and spin in core affect and interpersonal behaviour. For example, Moskowitz and Zuroff $(2004,2005 a)$ found that flux in quarrelsome behaviour was negatively related to Agreeableness; a similar relationship was reported by Kuppens et al. (2007) with regard to flux in the pleasure of core affect. Flux in submissiveness, like flux in arousal, appeared to be positively related to Neuroticism and Agreeableness. Past research is less clear regarding the associations with pulse. Finally, spin in both an interpersonal as well as a core affect space were positively related to Neuroticism, and negatively to Extraversion and Agreeableness.

In view of all this, the main goal of the present paper is to gain insight into the relationship between affect and behaviour dynamics, by examining whether there is a correspondence between individual differences in within-person variability in core affect on the one hand and in interpersonal behaviour on the other hand. First, we will try to replicate the findings of Moskowitz and Zuroff (2004, 2005a) and Kuppens et al. (2007) with respect to the relations of intraindividual variability in a core affect on the one hand and an interpersonal behaviour space on the other hand with several dispositional variables. More specifically, we aim to replicate the following findings of Kuppens et al. (2007): First, a positive relation between Extraversion and mean scores on pleasure and arousal; second,

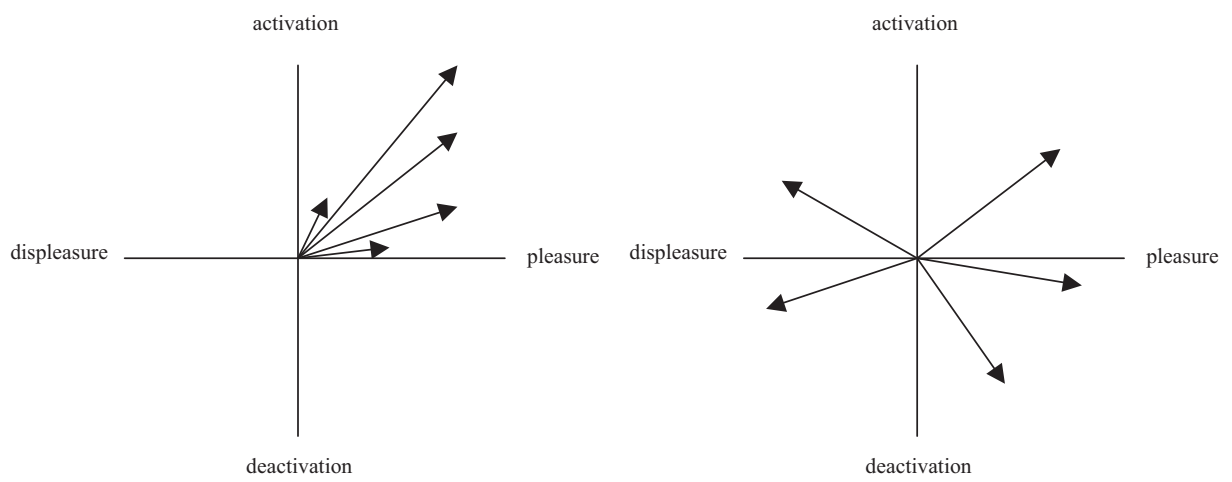

Figure 1. Graphical depictions of (a) high pulse (intensity variability) and low spin (quality variability) and (b) low pulse and high spin. 
these researchers found a positive relation between Conscientiousness and both mean scores, whereas this dispositional variable negatively correlated with spin. Agreeableness correlated negatively with flux arousal. And, finally, Kuppens et al. (2007) found a positive relation between Neuroticism and mean pleasure, as well as a positive relation with spin. Our predictions concerning the relation between intraindividual variability in an interpersonal behaviour circumplex and dispositional variables are based on the findings of Moskowitz and Zuroff (2004, 2005a). They found that a negative relation between Extraversion and spin; Agreeableness appeared to be positively related to mean communion as well as negatively to flux in communal behaviour and spin. Finally, Neuroticism displayed a negative relation with mean scores on communal and agentic behaviour and a positive relation with flux in agentic behaviour.

Second, and more importantly, we will investigate directly whether individual differences in variability (in terms of flux, pulse and spin) in both spaces relate to each other. Given the above, we will primarily investigate whether flux in pleasure is related to flux in communion, and whether flux in arousal is related to flux in agency. Apart from the fact that these characteristics showed similar relationships with dispositional variables, our predictions are based on previous theorizing that formulated specific hypotheses about how emotions may drive particular behaviours, in some cases even speculating that emotions are sufficient or necessary conditions for behaviours to appear. Berkowitz and Harmon-Jones (2004) identified negative mood as a sufficient condition for becoming aggressive, and Salovey and Rosenhan (1989) found that positive affect consistently promotes altruism and helping behaviour. Both suggest that flux pleasure and flux communion should be related. As to the relationship between arousal and agency, it has been argued that influencing others requires action, which involves increases in physiological arousal (such as heart rate, skin conductance, etc.), leading to the conclusion that influence should be associated with high arousal states (Tsai, 2007). It could therefore be expected that agentic behaviour and arousal may be related in people's feelings and behaviour (e.g. Mehrabian \& Russell, 1974). Furthermore, we will explore the relation between affective and interpersonal pulse and spin. Based on the above premises, we expect these to be mutually interrelated as well.

To answer these questions we performed a study in which participants reported on their core affect and interpersonal behaviour as experienced across multiple occasions, using an experience sampling method. Advantages of such a method include that people do not have to rely on their memories (which may eliminate cognitive biases of information storage and retrieval; e.g. Stone et al., 1998) and the high ecological validity because data-collection occurs within the real-life circumstances of the participant, rather than in artificial settings (Conner, Feldman Barrett, Tugade, \& Tennen, 2007; Feldman-Barrett \& Barrett, 2001; Hektner, Schmidt, \& Csikszentmihalyi, 2007). Data were collected during two consecutive weeks, with one week being devoted to measurements of core affect and another week to measurements of interpersonal behaviour.

\section{METHOD}

\section{Participants}

Participants were 63 students from the Katholieke Universiteit Leuven enrolled in multiple study programs. The sample consisted of 49 women and 14 men, with a mean age of 20.25 years $(S D=3.32$, range $=18-26)$. Thirty students were paid according to a variable 
payment scheme; other students participated in the study in fulfilment of class requirements. No significant differences were found between the paid and the nonpaid groups.

\section{Materials}

Core affect

Core affect was measured using the Affect Grid (Russell, Weiss, \& Mendelsohn, 1989), a visual $9 \times 9$ two-dimensional grid, with a neutral (fifth) row and a neutral (fifth) column. The vertical dimension of this grid was anchored at the top by highly active and at the bottom by sleepy, and the horizontal dimension on the left by unpleasant and on the right by pleasant; the corners (clockwise, starting from top right) were anchored by the words excited, relaxed, depressed and stressed.

\section{Interpersonal behaviour}

Interpersonal behaviour was measured using the Interpersonal Grid (Moskowitz \& Zuroff, 2005b), which is based on the interpersonal circumplex model of interpersonal behaviour. Again a $9 \times 9$ two-dimensional grid was used, with a neutral (fifth) row and a neutral (fifth) column. The vertical dimension was anchored at the top by assureddominant and at the bottom by unasserted-submissive, and the horizontal dimension on the left by cold-quarrelsome and on the right by warm-agreeable; the corners (clockwise, starting from top right) were anchored by the words outgoing, unassuming, aloof and arrogant.

\section{Personality questionnaire}

The Dutch version of the NEO-FFI (Hoekstra, Ormel, \& de Fruyt, 1996) was used to measure the dimensions of the Five Factor model of personality. The questionnaire consists of 60 items that constitute five 12-item scales assessing Neuroticism, Extraversion, Openness to Experience, Agreeableness and Conscientiousness. All items are to be rated on a 5 -point scale ranging from $1=$ strongly disagree to $5=$ strongly agree. Cronbach's $\alpha$ was .90 for Neuroticism, .73 for Extraversion, .66 for Openness to Experience, .76 for Agreeableness and .77 for Conscientiousness, indicating a satisfactory internal consistency.

\section{Procedure}

During an introductory session participants received information about the study and a programmed palmtop computer (Palm Tungsten E2). The palmtops were programmed with an adjusted version of the ESP software (developed by Feldman Barrett) that allowed administration of the Affect and Interpersonal Grid. It was explained that they would have to monitor their core affect during 1 week and their interpersonal behaviour during another one (with order of task being counterbalanced over participants). After participants had received this information, they were asked to complete the NEO-FFI questionnaire.

After the introductory session, participants received nine beeps a day during 14 consecutive days ( 9 beeps $\times 14$ days $=126$ beeps). The time of the first beep was prespecified on an individual basis with different times being allowed for weekdays and the weekend, to minimize the number of missed beeps (van Eck, Nicolson, \& Berkhof, 1998). Beeps during the day were programmed according to a stratified random interval scheme: 
The total amount of available daytime was divided into equal intervals, and a random beep was scheduled in each interval. During 1 week, participants were instructed to mark the position in the Affect Grid that best corresponded to how they felt at each sampling moment (signalled by a beep). During the other week, they were asked at each beep whether they had had an social interaction, defined as every social contact between individuals or groups, since the previous beep; if this was the case, they had to report on their interpersonal behaviour during their most recent interaction in terms of their position in the Interpersonal Grid. If the participant did not have an interaction since the last beep, filler items were presented.

At the end of the study, participants attended a second meeting during which they returned their palmtop computer and were paid (when applicable): Paid participants could earn $€ 25$ for compliance with the procedure and $\mathrm{a} € 1$ bonus for each day with 8 or 9 answered beeps; another $€ 1$ reward if this happened regularly, resulting in a maximum of $€ 40$.

\section{Analysis}

On the basis of the participants' positions on the Affect and Interpersonal Grids (coded in terms of Cartesian coordinate scores $(x, y)$ ranging from -4 to +4$)$ central tendency and variability measures were calculated per individual and per grid across all measurement occasions. We calculated within-person mean scores and standard deviations, called flux, on the dimensions of pleasure and arousal and communion and agency. Subsequently, on the basis of the framework of Moskowitz and Zuroff (2004), pulse (intensity variability) and spin (quality variability) measures were calculated both for the core affect and interpersonal behaviour scores (making use of transformations of the Cartesian coordinate scores $(x, y)$ into polar coordinates $(r, \theta))$. Pulse was calculated as the standard deviation of the $r$-coordinate values around the participant's mean $\left(r_{\mathrm{m}}\right)$, and as such represents how an individual fluctuates between more and less intense core affect (resp. interpersonal behaviour). Furthermore, spin was calculated as the circular standard deviation of the $\theta$-values, ${ }^{1}$ and as such represents the extent to which a person moves between qualitatively different angles in the core affect or interpersonal behaviour space.

Next, we calculated correlations between on the one hand the different mean and intraindividual variability scores (viz., means and flux of the scores on the constituent dimensions as well as affective and interpersonal pulse and spin), and on the other hand the Five Factor dimensions. Finally, the correlations between the different intraindividual characteristics of the respective circumplexes were calculated.

\section{RESULTS}

\section{Compliance with protocol}

For core affect, participants completed $86 \%$ of the occasions out of the total number of measurement occasions. For interpersonal behaviour, this was the case in $83 \%$ out of the total number of measurement occasions, including the occasions for which the participants

\footnotetext{
${ }^{1}$ Calculation of the circular standard deviation (Mardia, 1972) implies the conversion of all $(r, \theta)$ points to unit vectors, the resultant of which, $\mathbf{R}$, is subsequently calculated. The circular standard deviation then equals $\sqrt{-2 \ln (\|\mathbf{R}\| / n)}$, with $\|\mathbf{R}\|$ denoting the length of $\mathbf{R}$ and with $n$ denoting the number of data points for the individual under study.
} 
indicated that they did not have had an interaction (the latter being the case in $26 \%$ out of the $83 \%$ of the measurement occasions). This yielded a mean number of 52 observations per participant for core affect, and of 54 observations per participant for interpersonal behaviour. These results are good in comparison with similar studies (e.g. Hektner \& Csikszentmihalyi, 2002).

In addition we regressed the number of ESM trial on compliance, separately for the affect and interpersonal behaviour data. The results indicated a negative significant effect for number of trial (core affect: $B=-0.006, p<.01$, interpersonal behaviour: $B=-0.005$, $p<.01)$ meaning that the chances of complying with the procedure decreased with every trial.

\section{Descriptive statistics core affect and interpersonal behaviour and reliability}

Summary statistics for the core affect measures are presented in the upper part of Table 1. No differences were found due to the order of measurement (affect vs. behaviour first). Participants' mean core affect is pleasant $(M=1.11$, which is significantly greater than the scale midpoint of $0, t(62)=9.74, p<.001)$ and neutral in terms of implied arousal level $(M=0.08$, which does not significantly differ from the scale midpoint of $0, t(62)=0.85$, $n s)$. Regarding intraindividual variability, participants displayed more flux in arousal $(M=1.83)$ compared to flux in pleasantness $(M=1.69)(t(62)=-2.24, p<.05)$ meaning that individuals showed more variability on the vertical dimension compared to the horizontal one. We also found that participants showed more qualitative variability (i.e. spin) $(M=1.35)$ than quantitative variability (i.e. pulse) in core affect $(M=1.01)$ $(t(62)=-0.33, p<.001)$. This indicates that participants had a higher tendency to shift

Table 1. Means and standard deviations for core affect, interpersonal behavior and personality traits

\begin{tabular}{lcc}
\hline Variable & $M$ & $S D$ \\
\hline Core affect & & \\
$\quad$ Mean pleasantness & 1.11 & 0.91 \\
Mean arousal & 0.08 & 0.77 \\
Flux pleasantness & 1.69 & 0.41 \\
Flux arousal & 1.83 & 0.46 \\
Affective pulse & 1.01 & 0.20 \\
Affective spin & 1.35 & 0.32 \\
& & \\
Interpersonal behaviour & & 0.68 \\
Mean communion & 2.09 & 0.54 \\
Mean agency & 0.34 & 0.47 \\
Flux communion & 1.47 & 0.45 \\
Flux agency & 1.39 & 0.23 \\
Interpersonal pulse & 0.91 & 0.28 \\
Interpersonal spin & 1.30 & \\
Personality traits & & 0.41 \\
Extraversion & 3.71 & 0.44 \\
Agreeableness & 3.72 & 0.45 \\
Conscientiousness & 3.55 & 0.66 \\
Neuroticism & 2.83 & 0.42 \\
Openness to experience & 3.47 & \\
\hline
\end{tabular}


from one quadrant of the core affect circumplex to another, than that they experienced shifts in the intensity of their feelings.

The corresponding summary statistics for interpersonal behaviour can be found in the middle part of Table 1. Participants' mean interpersonal behaviour is agreeable $(M=2.09$, which does significantly differ from the scale midpoint of $0, t(62)=24.22, p<.001$ ), and mildly dominant $(M=0.34$, which is significantly greater than the scale midpoint of 0 , $t(62)=4.98, p<.001)$. Participants displayed an equal amount of flux in communal $(M=1.47)$ and agentic $(M=1.39)$ behaviour $(t(62)=1.47, n s)$. Regarding pulse and spin in interpersonal behaviour, we found that participants showed more interpersonal spin $(M=1.30)$ than pulse $(M=0.91)(t(62)=-0.40, p<.001)$. This means that participants had a higher tendency to shift between qualitatively different interpersonal behaviours than that they varied the intensity of their behaviours.

Regarding reliability, Spearman-Brown split-half reliability coefficients were calculated on the basis of data from the second, third and fourth day of sampling and data from the fifth, sixth and seventh day of sampling. These values equalled $0.70,0.68,0.43,0.80,0.44$ and 0.62 , for mean pleasantness, mean arousal, flux pleasantness, flux arousal, affective pulse and affective spin, respectively. The values for mean communion, mean agency, flux communion, flux agency, interpersonal pulse and interpersonal spin equalled $0.61,0.65$, $0.58,0.82,0.57$ and 0.64 , all suggesting acceptable reliability over time (taking into account the short time intervals of three days on which they are based).

\section{Relations between individual differences in core affect and personality traits}

In Table 2 the correlations between the mean and intraindividual variability scores for core affect and the Five Factor dimensions are presented. As appears from this table, the correlations are in general rather weak. The strongest associations are found for Neuroticism and Agreeableness: Highly neurotic individuals, compared to lowly neurotic ones, tend to feel less pleasant and display less flux on the arousal dimension; from their part, highly agreeable individuals, compared to lowly agreeable ones, display less flux on the arousal dimension, too. Despite the overall weaker correlations and lack of significance, the overall pattern of associations between intraperson variability scores in core affect and personality was similar to that reported by Kuppens et al. (2007). As evidence of this, the correlation between the Fisher's- $Z$-transformed correlations reported in Table 2 and those reported by Kuppens et al. (Study 1, 2007) was $0.54(p<.01)$. Results did not change when gender was statistically controlled.

Table 2. Correlations between core affect personality measures

\begin{tabular}{lcccrr}
\hline & $\mathrm{E}$ & $\mathrm{A}$ & $\mathrm{C}$ & $\mathrm{N}$ & $\mathrm{O}$ \\
\hline Mean pleasantness & 0.18 & 0.15 & 0.11 & $-0.26^{*}$ & -0.11 \\
Mean arousal & 0.18 & -0.02 & 0.22 & -0.14 & 0.07 \\
Flux pleasantness & 0.09 & -0.12 & 0.15 & -0.02 & 0.01 \\
Flux arousal & 0.10 & -0.22 & 0.12 & -0.24 & 0.03 \\
Affective pulse & -0.10 & 0.03 & 0.09 & -0.01 & -0.04 \\
Affective spin & -0.04 & -0.18 & 0.08 & 0.20 & 0.02 \\
\hline
\end{tabular}

Note: $N=63$.

E, Extraversion; A, Agreeableness; C, Conscientiousness; N, Neuroticism; O, Openness to Experience. ${ }^{*} p<.05$. 
Beyond the relations between the Five Factor dimensions and different constituent aspects of the core affect space, one may wonder how the personality dimensions can be situated within the core affect space as a whole. To answer this question we projected each of the Five Factor dimensions in the core affect space by means of multiple regression analyses in which one Five Factor dimension served as a criterion and the intraindividual mean scores on the two core affect dimensions as predictors. The results are displayed in the left panel of Figure 2. Neuroticism shows the strongest relationship with the core affect space and is positioned fairly closely to the negative pole of pleasure, meaning that highly neurotic individuals tend to experience on average more negative affect (compared to lowly neurotic ones). Highly conscientious or extraverted individuals (compared to lowly conscientious and extraverted ones) tend to experience on average more positive affect and feel highly activated. Multiple regression analyses showed weak relationships between the core affect dimensions and Openness to Experience and Agreeableness.

\section{Relations between individual differences in interpersonal behaviour and personality traits}

The correlations between mean and intraindividual variability scores for interpersonal behaviour and the Five Factor dimensions are reported in Table 3. Again, the correlations are in general rather weak, albeit somewhat stronger than for core affect. The highest correlations now were found for Extraversion and Agreeableness: Highly agreeable individuals, compared to lowly agreeable ones, behave in a more agreeable way towards their partners, tend to display less flux with respect to communal behaviours, and show less spin in their interpersonal behaviour; from their part, highly extraverted individuals, compared to lowly extraverted ones, tend to behave in a more agentic way towards their interaction partners, and with less qualitative variability (i.e. spin). Results did not change when gender was statistically controlled.

We also investigated how the different personality dimensions can be situated in the interpersonal space as a whole. The results of this projection are displayed in the right panel
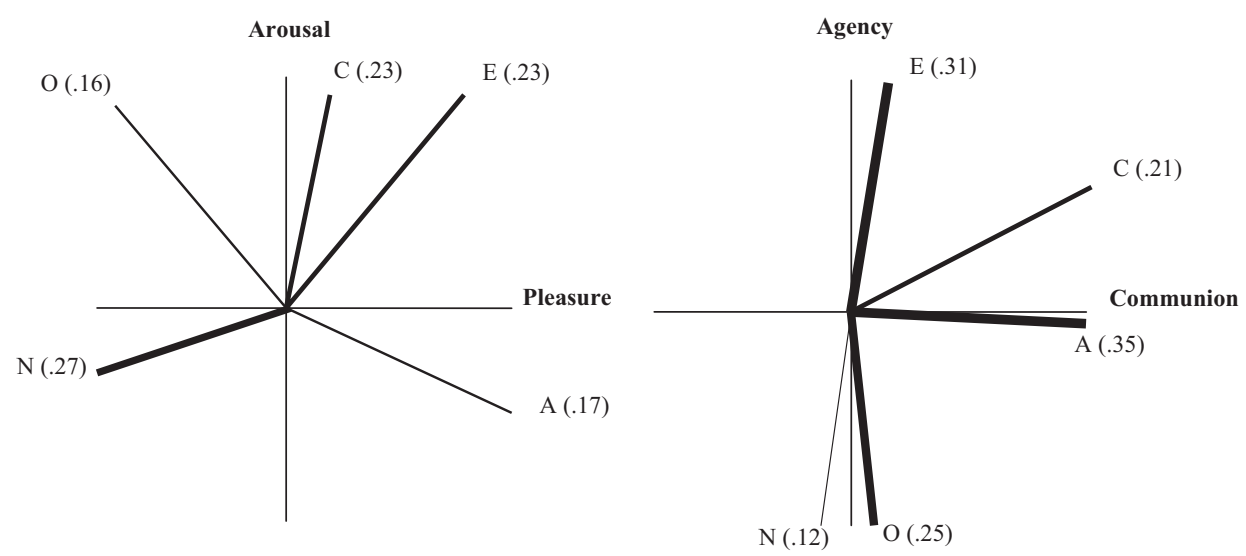

Figure 2. Multiple regression of the core affect (left) and the interpersonal behaviour dimensions (right) on the Five Factor personality variables. The multiple correlation coefficients are indicated between parentheses and are also depicted by the thickness of the projected Big Five Dimensions.

Note: E, Extraversion; A, Agreeableness; C, Conscientiousness; N, Neuroticism; O, Openness to Experience. 
Table 3. Correlations between interpersonal behaviour and personality measures

\begin{tabular}{lccrrr}
\hline & $\mathrm{E}$ & $\mathrm{A}$ & $\mathrm{C}$ & $\mathrm{N}$ & \multicolumn{1}{c}{$\mathrm{O}$} \\
\hline Mean Communion & 0.03 & $0.35^{* *}$ & 0.20 & 0.01 & \multicolumn{1}{c}{0.03} \\
Mean Agency & $0.30^{*}$ & -0.04 & 0.05 & -0.12 & $-0.25^{*}$ \\
Flux Communion & -0.17 & $-0.27^{*}$ & -0.02 & -0.12 & -0.04 \\
Flux Agency & 0.01 & -0.10 & -0.01 & -0.14 & 0.05 \\
Interpersonal Pulse & -0.14 & -0.11 & 0.02 & 0.01 & 0.02 \\
Interpersonal Spin & $-0.31^{*}$ & $-0.44^{* *}$ & -0.20 & 0.12 & -0.02 \\
\hline
\end{tabular}

Note: $N=63$.

E, Extraversion; A, Agreeableness; C, Conscientiousness; N, Neuroticism; O, Openness to Experience. ${ }^{*} p<.05 ;{ }^{* *} p<.01 ;{ }^{* * *} p<.001$.

of Figure 2. Extraversion and Agreeableness show the strongest relationships with the interpersonal behaviour space. Furthermore, Extraversion appears to be closely related to mean agency, whereas trait Agreeableness almost coincides with mean communion. We found a less strong relationship between Openness to Experience and the interpersonal circumplex, indicating that highly open individuals (compared to lowly ones) tend to display on average more submissive behaviour, and between Conscientiousness and interpersonal behaviour space, indicating that highly conscientious individuals (compared to lowly ones) show on average more agreeable behaviour.

\section{Relations between individual differences in core affect and interpersonal behaviour}

In Table 4, the correlations between the different mean and intraindividual variability scores in core affect and their interpersonal counterparts are presented. No differences were found due to the order of measurement (affect vs. behaviour first). We predicted a more or less one-to-one relation in terms of strong correlations on the diagonal of this table. All correlations on this diagonal are positive, with most of them being significant and of moderate size. Regarding the correlations between the constituent dimensions of the two spaces, the strongest associations were found for arousal and agentic behaviour; the situation is less clear for pleasure and communion, as flux in the horizontal dimension in each space appears to be related to flux in both the horizontal and the vertical dimension of the other space. As regards the quantitative and qualitative variability measures pulse and

Table 4. Correlations between individual differences in core affect and interpersonal behaviour measures

\begin{tabular}{lcccccc}
\hline & $\begin{array}{c}\text { Mean } \\
\text { pleasantness }\end{array}$ & $\begin{array}{c}\text { Mean } \\
\text { arousal }\end{array}$ & $\begin{array}{c}\text { Flux } \\
\text { pleasantness }\end{array}$ & $\begin{array}{c}\text { Flux } \\
\text { arousal }\end{array}$ & $\begin{array}{c}\text { Affective } \\
\text { pulse }\end{array}$ & $\begin{array}{c}\text { Affective } \\
\text { spin }\end{array}$ \\
\hline Mean communion & 0.21 & -0.12 & 0.10 & 0.08 & 0.19 & -0.12 \\
Mean agency & 0.25 & $0.40^{* *}$ & $-0.27^{*}$ & -0.01 & -0.06 & -0.10 \\
Flux communion & -0.04 & $-0.28^{*}$ & $0.43^{* * *}$ & $0.41^{* *}$ & $0.27^{*}$ & $0.28^{*}$ \\
Flux agency & 0.04 & $-0.26^{*}$ & $0.44^{* * *}$ & $0.50^{* * *}$ & 0.11 & 0.19 \\
Interpersonal pulse & -0.16 & -0.06 & 0.13 & 0.12 & 0.24 & 0.23 \\
Interpersonal spin & -0.20 & -0.14 & 0.13 & $0.29^{*}$ & 0.15 & $0.36^{* *}$ \\
\hline
\end{tabular}

Note: $N=63$.

${ }^{*} p<.05 ;{ }^{* *} p<.01 ;{ }^{* * *} p<.001$. 
spin, a modest correlation was found for the former and a moderately high one for the latter. Results did not change when gender was statistically controlled.

To examine whether the correlations between the flux measures were due to their variability component and not to their associations with their mean counterparts, we calculated partial correlations between flux pleasantness and flux communion, controlling for mean pleasantness and mean communion, and between flux arousal and flux agency, controlling for mean arousal and mean agency. These correlations equalled 0.55 and 0.46 (both $p s<.001$ ), clearly indicating that the obtained correlations between the flux measures reflect associations between affective and behavioural variability (and are not due to their associations with the means on their respective dimensions).

\section{DISCUSSION}

\section{Relations between individual differences in core affect and interpersonal behaviour and personality traits}

Regarding relations between intraperson variability in core affect and personality traits, we wanted to check whether we could replicate findings of Kuppens et al. (2007) on these relations. First, the associations found in our study were of a more modest nature than those of Kuppens et al. (2007). Nevertheless, the relations that showed up in our study showed a very similar pattern, as evidenced by the high correlation between the correlation patterns from the two studies. Inspection of the standard deviations of the variables under study (Table 1) suggests that the difference in size of the correlation between both studies is not to be attributed to restriction of range. The most straightforward explanation for this difference in magnitude, may lie in a difference in reliability of the core affect variability measures. The compliance in the Kuppens et al. study was substantially higher (on average, participants provided reports in 60 of the 63 programmed beeps, whereas in the current study participants on average reported in response to 52 of the 63 programmed). As a result, the measures reported in the current study were based on fewer measurement occasions, affecting their reliability and consequently lowered their correlations with other measures.

The single significant correlation in the present study, indicated that highly neurotic individuals have in general lower pleasure scores, a finding that replicates previous results from Larsen and Ketelaar (1991). The finding that the level of flux in pleasantness is similar for lowly and highly neurotic individuals and that highly neurotic individuals do not show more flux in arousal was unexpected. And despite evidence from previous studies (e.g. Lucas \& Baird, 2004), the correlation between mean pleasantness and Extraversion also appeared to be non-significant in the present study.

Second, we investigated whether we could replicate the results of Moskowitz and Zuroff $(2004,2005 a)$ on the relations between individual differences in intraperson variability in interpersonal behaviour and personality traits. In general, all significant correlations with Extraversion and Agreeableness in the study of Moskowitz and Zuroff (2004, 2005a) also appeared to be significant in the present study. In particular, we found that agreeable individuals tend to display more communion-type behaviour, and less variability in communion and spin; in their turn, extraverted individuals tend to display more dominant behaviour, and less variability in spin. As regards the relationships with Neuroticism, we did not replicate the findings of Moskowitz and Zuroff (2004, 2005a), as no significant correlations with Neuroticism were found in the present study. Inspection of the standard 
deviation of the Neuroticism variable (Table 1) suggests that this is not to be attributed to restriction of range.

Third, beyond the relations between the Five Factor dimensions and several indices of intraindividual variability per circumplex, we also examined the relations between the Five Factor dimensions and the mean positions in the two spaces as a whole. From the projections of the Five Factor dimensions into the two spaces, it appeared that Neuroticism bears the closest relationship with core affect, and Extraversion and Agreeableness the closest relationship with interpersonal behaviour (Schmidt, Wagner, \& Kiesler, 1999; Wiggins \& Broughton, 1991; Wiggins \& Pincus, 1994). Indeed, these findings directly reflect the meaning or nature of the respective Five Factor dimensions and highlight that Neuroticism as a trait measuring emotional lability (e.g. Eysenck, 1991) most strongly relates to affect, whereas Extraversion and Agreeableness are intrinsically interpersonal in nature (McCrae \& Costa, 1989).

\section{Relations between individual differences in core affect and interpersonal behaviour}

The main goal of the present paper was to investigate in a direct way whether there is indeed a link between individual differences in intraperson variability scores in core affect on the one hand and in interpersonal behaviour on the other hand. Primarily we looked at the relation between individual's mean positions in the core affect and interpersonal behaviour space. The results displayed a clear correspondence between the mean scores in the arousal and agency dimensions of the two spaces whereas the correspondence was rather weak for the pleasure and communion dimension. This difference is echoed at the level of flux in that the correspondence between flux measures of the vertical dimensions is somewhat stronger than that between the horizontal dimensions.

These findings on the vertical dimensions support the hypothesis that a higher level of arousal is involved in agentic behaviours (Tsai, 2007). This is also in line with the study of Galinsky, Gruenfeld and Magee (2003), which showed that individuals who were put in highly dominant positions or who were instructed to recall moments at which they felt powerful, were likely to experience more arousal, than those who were not.

The finding that there is a weak correspondence only between pleasure and communion further lines up with the inconsistent results from research at this point: On the one hand, Berkowitz and Harmon-Jones (2004) and Salovey and Rosenhan (1989) reported evidence for a correspondence between variability in how pleasant one feels and how agreeable one behaves towards others; on the other hand, Anderson, Langner and Keltner (2001) found that highly dominant men reported elevated positive mood levels prior to completing experimental tasks.

The obtained correspondence between core affect and interpersonal behaviour also suggests that the relation between both the two spaces is more complex than the one-to-one relation between the horizontal and vertical dimensions we initially assumed. To check whether a more complex relation between both spaces would hold indeed, we projected the interpersonal space in the core affect space (making use of multiple regression analyses in which the intraindividual mean score on one interpersonal dimension served as a criterion and the mean scores on the two core affect dimensions as predictors). The results of these projections are displayed in Figure 3, with thickness of the projected interpersonal dimensions reflecting the size of the corresponding multiple correlations. From this figure, it appears that agency fits better in the core affect space than communion. Furthermore, the 


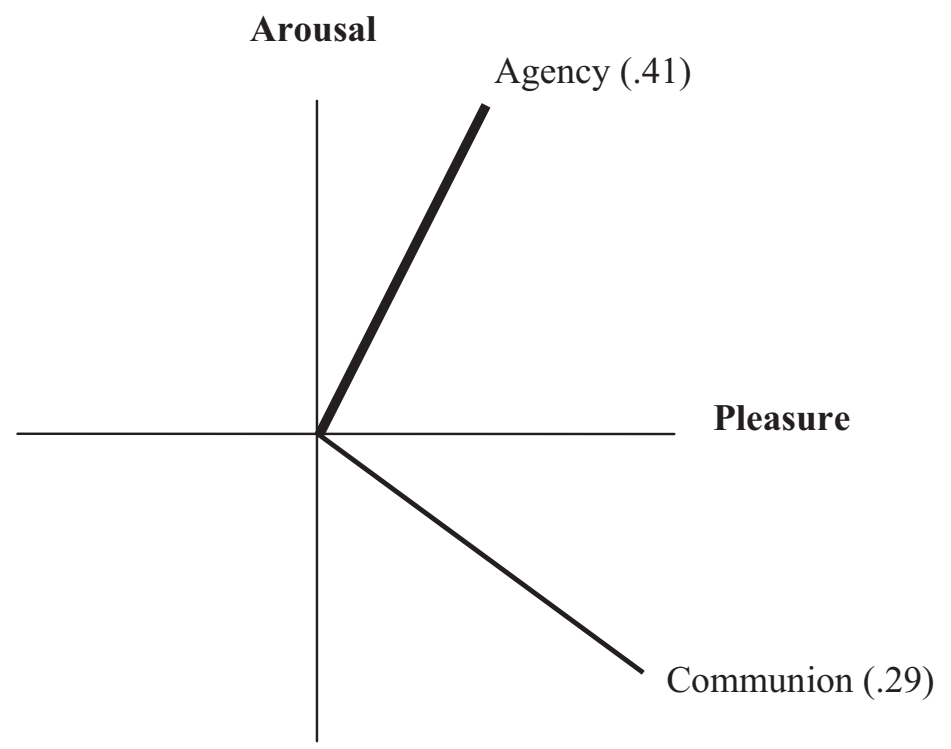

Figure 3. Multiple regression of the core affect dimensions on the interpersonal behaviour dimensions with the multiple correlation coefficients between parentheses. The thickness of the projected interpersonal dimensions reflects the size of the corresponding correlation coefficients.

findings show that agency maps on fairly purely to arousal, whereas communion should more be regarded as relating to a combination of pleasure and low arousal. The latter deviation also reflects that quarrelsome individuals are characterized by combatively higher arousal levels as compared to friendly individuals, echoing findings from research on the hypothesis of transfer of excitation to explain aggressive behaviour (Zillmann, 1971). In any case, our findings show that the affective and behavioural circumplex do not perfectly map on to each other in terms of how people behave and feel on average.

Our findings indicate a strong correspondence between the flux measures of both spaces: flux pleasantness and arousal show strong relationships with flux communion as well as with agency. Furthermore, we also found that individuals with a strong tendency to experience qualitatively different emotions (as captured by high levels of spin) tend to behave in qualitatively different ways towards their interaction partners. All this implies clear evidence for the pervasive nature of instability. In general, individuals that are more variable in terms of core affect also display more volatile interpersonal behaviour. At this point, it is not clear however how the direction of this relationship should be interpreted, but both directions appear valid. Core affect variability may signal insecurity, low selfesteem and psychological maladjustment (Kuppens et al., 2007); this may cause people to be less firm and more susceptible to the behaviour of their interaction partners when engaging in interpersonal contacts, causing them to show higher behavioural variability than emotionally less variable individuals. Conversely, it is also conceivable that high behavioural variability promotes emotional variability: An individual characterized by high behavioural variability will go through more diverse interpersonal encounters in terms of status and communion, which in turn may cause higher emotional volatility as well.

Obviously, there are a number of possible limitations to this study. First, our sample was imbalanced with more women than men. Although some gender differences may have been expected, for example in the relationship between agency and pleasure, the results did not 
change when controlling for gender. Another limitation is that since participants were instructed to mark their core affect and their interpersonal behaviour during different weeks, one could argue that completing both questionnaires at the same time would have resulted in more accurate data. However, because our main focus was to relate individual differences in intraindividual variability in core affect and interpersonal behaviour, and previous studies have shown that these individual differences are temporally stable (Eaton \& Funder, 2001; Moskowitz \& Zuroff, 2005a), there was no need to collect the two types of data at the same time. Moreover, asking participants to mark their position on the Affect Grid and the Interpersonal Grid at the same time may have caused interference between both measurements (e.g. in terms of unwanted demand characteristics).

Another limitation pertains to the possibility of memory biases with regard to the interpersonal behaviour ratings that were assessed. This effect can be complicated by the fact that not all social interactions are the same with regard to quality or intensity. Nevertheless, by using an experience sampling method with a maximum time interval of two hours, we minimized this.

A final possible limitation pertains to the fact that a graphical single-item scale was used to measure affect and behaviour. We choose this scale because of multiple reasons. A first reason was the very economic way of measurement, which is a crucial consideration when using an experience sampling method. The second reason was that previous research has shown that both scales have adequate reliability, convergent validity and discriminant validity (e.g. Moskowitz \& Zuroff, 2005b; Russell et al., 1989).

In conclusion, the present study presents evidence that how people's feelings and interpersonal behaviour change across time are interrelated. The results demonstrated a clear correspondence between several indices of intraindividual variability in arousal and agency, and also between intraindividual variability in valence and communion, albeit the latter was of smaller magnitude. These results point to the pervasive nature of instability, with high affective variability coinciding with high behavioural variability. However, the finding that agency seemed to fit better in the core affect circumplex than communion, suggests that the affective and behavioural circumplex do not perfectly map on to each other.

\section{REFERENCES}

Anderson, C., Langner, C., \& Keltner, D. (2001). Status, power, and emotion. Unpublished manuscript.

Barrett, K. C. (1998). A functionalist perspective to the development of emotions. In M. F. Mascolo, \& S. Griffin (Eds.), What develops in emotional development? (pp. 109-133). New York: Plenum Press.

Berkowitz, L., \& Harmon-Jones, E. (2004). Toward an understanding of the determinants of anger. Emotion, 4, 107-130.

Conner, T. S., Feldman Barrett, L., Tugade, M. M., \& Tennen, H. (2007). Idiographic personality: The theory and practice of experience sampling. In R. W. Robins, R. C. Fraley, \& R. F. Krueger (Eds.), Handbook of research methods in personality psychology (pp. 79-98). New York, NY: Guilford Press.

Eaton, L. G., \& Funder, D. C. (2001). Emotional experience in daily life: Valence, variability, and rate of change. Emotion, 1, 413-421.

Eysenck, H. J. (1991). Dimensions of personality: 16, 5 or 3? Criteria for a taxonomic paradigm. Personality and Individual Differences, 12, 773-790. 
Feldman-Barrett, L., \& Barrett, D. J. (2001). Computerized experience-sampling: How technology facilitates the study of conscious experience. Social Science Computer Review, 19, 175-185.

Frijda, N. H. (1986). The emotions. Cambridge: Cambridge University Press.

Frijda, N. H., \& Mesquita, B. (1994). The social roles and functions of emotions. In S. Kitayama, \& H. R. Markus (Eds.), Culture and emotion (pp. 51-88). Washington, DC: American Psychological Association.

Galinsky, A. D., Gruenfeld, D. H., \& Magee, J. C. (2003). From power to action. Journal of Personality and Social Psychology, 85, 453-466.

Hektner, J. M., \& Csikszentmihalyi, M. (2002). The experience sampling method: Measuring the context and content of loves. In R. B. Bechtel, \& A. Churchman (Eds.), Handbook of environmental psychology (pp. 233-243) NY: John Wiley \& Sons.

Hektner, J. M., Schmidt, J. A., \& Csikszentmihalyi, M. (2007). Experience sampling method: Measuring the quality of everyday life. Thousand Oaks, CA: Sage.

Hoekstra, H. A., Ormel, J., \& De Fruyt, F. (1996). NEO PI-R, NEO FFI Big Five Persoonlijkheidsvragenlijsten: Handleiding. Lisse, The Netherlands: Swets \& Zeitlinger B.V.

Kuppens, P., Van Mechelen, I., Nezlek, J. B., Dossche, D., \& Timmermans, T. (2007). Individual differences in core affect variability and their relationship to personality and psychological adjustment. Emotion, 7, 262-274.

Lucas, R. E., \& Baird, B. M. (2004). Extraversion and emotional reactivity. Journal of Personality and Social Psychology, 86, 473-485.

Larsen, R. J., \& Ketelaar, T. (1991). Personality and susceptibility to positive and negative emotional states. Journal of Personality and Social Psychology, 61, 132-140.

Mardia, K. V. (1972). Statistics of directional data. New York: Academic Press.

McCrae, R. R., \& Costa, P. T., Jr. (1989). The structure of interpersonal traits: Wiggins's circumplex and the five-factor model. Journal of Personality and Social Psychology, 56, 586-595.

Mehrabian, A., \& Russell, J. A. (1974). An approach to environmental psychology. Boston: MIT Press.

Moskowitz, D. S., \& Zuroff, D. C. (2004). Flux, pulse, and spin: Dynamic additions to the personality lexicon. Journal of Personality and Social Psychology, 86, 880-893.

Moskowitz, D. S., \& Zuroff, D. C. (2005a). Robust predictors of flux, pulse and spin. Journal of Research in Personality, 39, 130-147.

Moskowitz, D. S., \& Zuroff, D. C. (2005b). Assessing interpersonal perceptions using the interpersonal grid. Psychological Assessment, 17, 218-230.

Niedenthal, P. M. (2007). Embodying emotion. Science, 316, 1002-1005.

Russell, J. A. (2003). Core affect and the psychological construction of emotion. Psychological Review, 110, 145-172.

Russell, J. A., Weiss, A., \& Mendelsohn, G. A. (1989). Affect grid: A single-item scale of pleasure and arousal. Journal of Personality and Social Psychology, 57, 493-502.

Salovey, P., \& Rosenhan, D. L. (1989). Mood states and prosocial behavior. In H. Wagner, \& A. Manstead (Eds.), Handbook of psychophysiology (pp. 371-391). New York: Wiley.

Scherer, K. R. (2009). The dynamic architecture of emotion: Evidence for the component process model. Cognition and Emotion, 23, 1307-1351.

Schmidt, J. A., Wagner, C. C., \& Kiesler, D. J. (1999). Covert reactions to big five personality traits: The impact message inventory and the NEO-PI-R. European Journal of Psychological Assessment, $15,221-232$.

Schimmack, U., \& Grob, A. (2000). Dimensional models of core affect: A quantitative comparison by means of structural equation modeling. European Journal of Personality, 14, 325-345.

Stone, A., Schwarz, J., Neale, J., Shiffman, S., Marco, C., Hickcox, M., et al. (1998). A comparison of coping assessed by ecologic momentary assessment and retrospective recall. Journal of Personality and Social Psychology, 74, 1670-1680.

Tsai, J. L. (2007). Ideal affect: Cultural causes and behavioral consequences. Perspectives on Psychological Science, 2, 242-259.

van Eck, M., Nicolson, N. A., \& Berkhof, J. (1998). Effects of stressful daily events on mood states: Relationship to global perceived stress. Journal of Personality and Social Psychology, $75,1572-1585$.

Wiggins, J. S. (1979). A psychological taxonomy of trait descriptive terms: The interpersonal domain. Journal of Personality and Social Psychology, 37, 395-412. 
Wiggins, J. S. (1991). Agency and communion as conceptual coordinates for the understanding and measurement of interpersonal behavior. In W. M. Grove, \& D. Cicchetti (Eds.), Thinking clearly about psychology (pp. 89-113). Minneapolis: University of Minnesota Press.

Wiggins, J. S., \& Broughton, R. (1991). A geometric taxonomy of personality scales. European Journal of Personality, 5, 343-365.

Wiggins, J. S., \& Pincus, A. L. (1994). Personality structure and the structure of personality disorders. In P. T. Costa, Jr., \& T. A. Widiger (Eds.), Personality disorders and the five-factor model of personality (pp. 73-93). Washington, DC: American Psychological Association.

Zillmann, D. (1971). Excitation transfer in communication-mediated aggressive behavior. Journal of Experimental Social Psychology, 7, 419-434. 